\title{
Espessura central da córnea e a medida da pressão intra-ocular com diferentes tonômetros
}

\section{Could the central cornea thickness change the intraocular pressure results obtained by different tonometers}

Priscila Yumi Kitice', Thiago Henrique Carvalho Nunes' ${ }^{2}$ Eduardo Della Giustina ${ }^{3}$, Rodrigo Andrade de Barros ${ }^{4}$, Walter Gomes Amorin Filho ${ }^{5}$, José Ricardo Carvalho Lima Rehder ${ }^{6}$

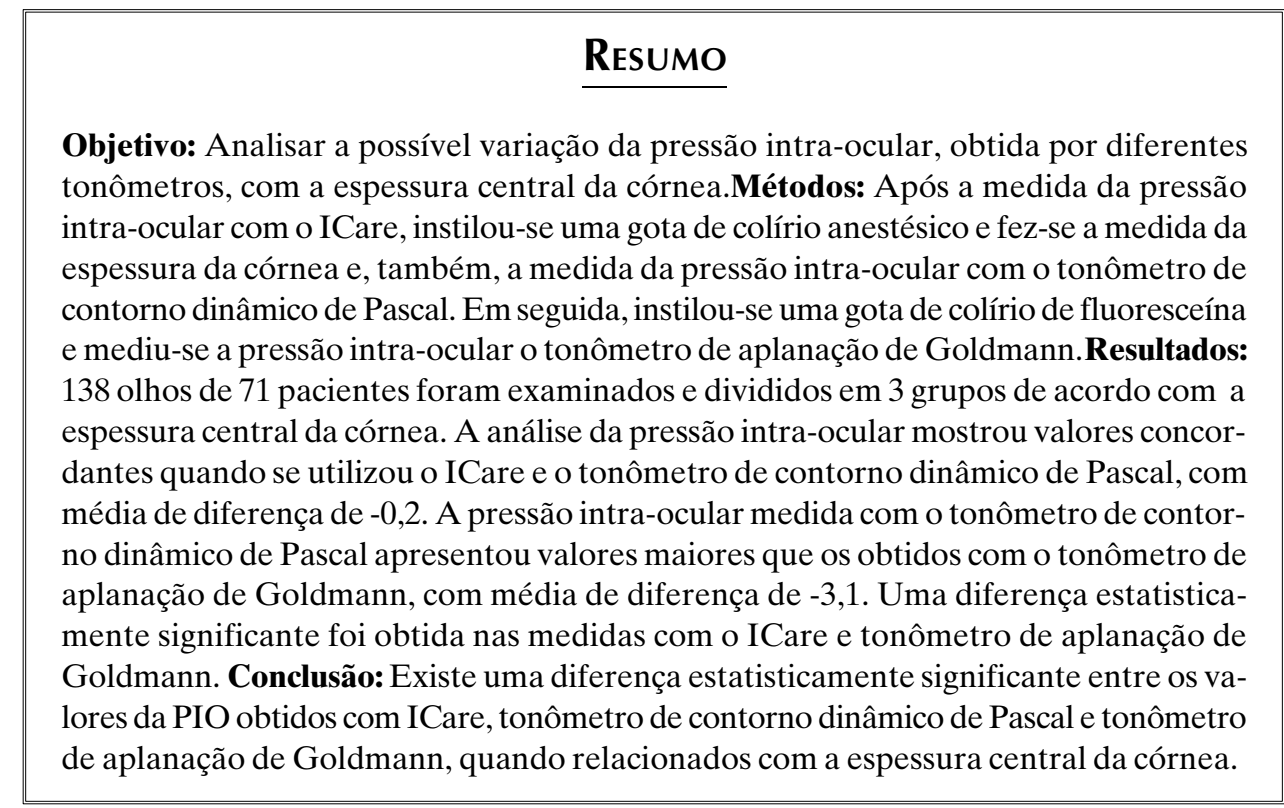

Descritores: Tanometria ocular/métodos; Córnea/anatomia \& histologia; Topografia da córnea; Glaucoma; Pressão intra-ocular

\footnotetext{
1 Fellow Glaucoma da Faculdade de Medicina do ABC - FMABC - Santo André (SP), Brasil;

${ }^{2}$ Fellow Glaucoma da Faculdade de Medicina do ABC - FMABC - Santo André (SP), Brasil;

3 Fellow Glaucoma da Universidade de São Paulo - USP - São Paulo (SP), Brasil;

${ }^{4}$ Médico colaborador do Setor de Glaucoma da Faculdade de Medicina do ABC - FMABC - Santo André (SP), Brasil;

${ }^{5}$ Chefe do Setor de Glaucoma da Faculdade de Medicina do ABC - FMABC - Santo André (SP), Brasil;

${ }^{6}$ Professor Titular de Oftalmologia da Faculdade de Medicina do ABC - FMABC - Santo André (SP), Brasil.
}

Trabalho realizado na Faculdade de Medicina do ABC - FMABC - Santo André (SP), Brasil.

Recebido para publicação em: 29/01/2007 - Aceito para publicação em 18/12/2007 


\section{INTRODUÇÃO}

$\mathbf{A}$ pressão intra-ocular (PIO) é um fator de risco importante no diagnóstico, progressão e tratamento do glaucoma, embora os valores obtidos apresentem uma baixa sensibilidade e uma baixa especificidade ${ }^{(1-4)}$.

A determinação precisa da PIO é um importante passo na detecção e seguimento do glaucoma, primário ou secundário, bem como no diagnóstico diferencial de glaucoma primário de ângulo aberto, glaucoma de pressão normal e hipertensão ocular ${ }^{(3,5)}$.

A PIO "normal" pode ser definida como a pressão que não causa dano na cabeça do nervo óptico, independentemente de seu valor numérico ${ }^{(1)}$.

Em 1955, Goldmann foi o primeiro a aplicar com sucesso o princípio da aplanação da córnea na tonometria. O tonômetro de aplanação de Goldmann (TAG) é desde então o método mais utilizado ("Gold Standard") para medir a PIO, porém com limitações relacionadas a espessura central da córnea (ECC), curvatura corneana, com a necessidade de instilação tópica de colírio anestésico e colírio de fluoresceína. Atualmente, as propriedades biomecânicas da córnea estão sendo consideradas também como importantes e possíveis fatores relacionados às variações da PIO entre diferentes individuos ${ }^{(2,47)}$

O ICare ${ }^{\varpi}$ (IC - Rebound tonometry, Tiolat Oy, Helsinki, Finland) afere a PIO através do método de "rebote". Após a formação de um campo magnético, uma sonda é acelerada em direção à córnea e um microprocessador analisa os parâmetros de movimento da sonda após a colisão. O IC tem como vantagens ser um tonômetro fácil de usar, portátil e não requer uso de anestesia tópica ou colírio de fluoresceína ${ }^{(1,5-6,8)}$.

O tonômetro de contorno dinâmico de Pascal ${ }^{\varpi}$ (TCDP - Swiss Microtechnology AG, Port, Switzerland) surgiu recentemente como um método para medir a PIO, supostamente independente das propriedades corneanas $^{(3,7-8)}$. O TCDP utiliza um sensor de pressão de cristal piezoelétrico, embutido no centro da superfície de uma ponteira delineada e côncava. Esta ponteira, quando em contato com a superfície corneana, mede a PIO sem distorções, uma vez que, não exerce nenhuma pressão sobre a córnea. Um microprocessador mede as variações da pressão intra-ocular durante a sístole e a diástole, apresentando as flutuações da PIO relacionadas com as pulsações cardíacas (amplitude de pulso - APO) ${ }^{(3,7)}$.

\section{ОвJetivo}

O objetivo deste estudo é comparar os valores de pressão intra-ocular obtidos com o IC, o TAG e o TCDP e correlacioná-los com a espessura central da córnea.

\section{Métodos}

Foram examinados, prospectivamente, 138 olhos de 71 pacientes não glaucomatosos, atendidos no ambulatório de oftalmologia da faculdade de medicina do ABC, de março a agosto de 2006, sendo 40 do sexo feminino e 31 do sexo masculino. Destes, 32 eram da raça negra e 39 caucasianos. A média de idade foi de 47,3 anos.

Após assinarem consentimento livre e esclarecido os pacientes foram submetidos a um exame oftalmológico completo.

Os pacientes foram divididos em 3 grupos de acordo com a ECC. Inicialmente, a PIO foi medida com o IC, sem anestesia tópica.

O ICare ${ }^{\circledast}$ pesa $26.5 \mathrm{mg}$, utiliza uma sonda descartável de aço inoxidável com ponteira de plástico de $0.9 \mathrm{~mm}$ de raio. Para medir a PIO, o aparelho é posicionado próximo ao olho do paciente e a ponta da sonda é mantida a uma distância de 4 a $8 \mathrm{~mm}$ da córnea. Através da formação de um campo magnético, a sonda é acelerada em direção à córnea a uma velocidade de $0.25-0.35 \mathrm{~m} / \mathrm{s}$. Um microprocessador analisa os parâmetros de movimento da sonda após a colisão. O IC é capaz de detectar possíveis erros durante a medida e descartar medidas que estejam fora do desvio padrão. Após seis medidas de boa qualidade, o maior e menor valor são descartados automaticamente e o valor da PIO é mostrado no display do aparelho, resultado da média das medidas consideradas ${ }^{(1,5-6)}$.

Após instilação, em ambos os olhos, de uma gota de colírio anestésico (tetracaína 1\%), utilizando-se um paquímetro ultra-sônico da marca Mentor (Mentor Ophthalmics Inc, Norwell, MA, USA) foi medida a ECC.

Em seguida, a PIO era medida com o TCDP. A qualidade da medida é classificada de 1 (ótimo) a 5 (inaceitável). Foram incluídas todas as medidas de qualidades 1 e 2 , embora a medida com qualidade 3 seja considerada aceitável. Após a instilação de uma gota de colírio de fluoresceína era medida a PIO com o TAG. As medidas da PIO foram realizadas com um intervalo de $5 \mathrm{mi}-$ nutos entre as técnicas.

Foram excluídos do estudo, pacientes com acuidade visual com a melhor correção óptica pior que 
20/40, glaucoma primário de ângulo aberto, glaucoma secundários, glaucoma primário de ângulo fechado, patologias corneanas e cirurgias intra-oculares prévias.

Os critérios de inclusão foram: pacientes sem glaucoma, com melhor visão corrigida 20/40, sem cirurgias oculares prévias. O trabalho foi analisado e aprovado pela Comissão de Ética em Pesquisa da Faculdade de Medicina do ABC

\section{Resultados}

Um total de 138 olhos de 71 pacientes foi avaliado. 40 pacientes do sexo feminino e 31 pacientes do sexo masculino, sendo 32 da raça negra e 39 caucasianos. A média de idade encontrada foi 47.3 anos.
A PIO média medida com o TAG foi $14.1 \mathrm{mmHg}$ $( \pm 3.2), 17.3 \mathrm{mmHg}( \pm 2.4)$ com o TCDP e $17.1 \mathrm{mmHg}$ $( \pm 4.0)$ com o IC.

Para correlacionar as medidas da ECC com os valores da PIO obtidos pelos diferentes tonômetros, os pacientes foram subdivididos em 3 grupos:

- Grupo 1: ECC menor que $520 \mu \mathrm{m}$ (48 olhos), média de $496.3 \mu \mathrm{m}( \pm 17.4 \mu \mathrm{m})$;

- Grupo 2: ECC entre 520-560 um (48 olhos), média de $542.4 \mu \mathrm{m}( \pm 10.3 \mu \mathrm{m})$;

- Grupo 3: ECC maior que $560 \mu \mathrm{m}$ (42 olhos), média de $578.8 \mu \mathrm{m}( \pm 15.8 \mu \mathrm{m})$.

A PIO média obtida pelos tonômetros nos grupos 1,2 e 3 está demonstrada na tabela 1 .

Foi estudada a concordância entre os métodos,

Tabela 1

Medida das pressões intra-oculares nos diversos grupos e com os diferentes tonômetros

\begin{tabular}{lccc}
\hline ECC & ICare $^{\circledast}$ & Goldmann & Pascal $^{\circledR}$ \\
\hline Grupo 1 & $15.7 \mathrm{mmHg}( \pm 3.9)$ & $13.7 \mathrm{mmHg}( \pm 3.3)$ & $17.3 \mathrm{mmHg}( \pm 2.6)$ \\
Grupo 2 & $16.3 \mathrm{mmHg}( \pm 3.0)$ & $13.8 \mathrm{mmHg}( \pm 2.5)$ & $17.2 \mathrm{mmHg}( \pm 2.0)$ \\
Grupo 3 & $19.4 \mathrm{mmHg}( \pm 3.9)$ & $14.9 \mathrm{mmHg}( \pm 3.5)$ & $17.1 \mathrm{mmHg}( \pm 2.3)$ \\
\hline
\end{tabular}

Tabela 2

Análise do coeficiente de concordância de Lin (Rc) TAGxIC

\begin{tabular}{lccc}
\hline & Grupo 1 & Grupo 2 & Grupo 3 \\
\hline $\mathrm{R}_{\mathrm{c}}$ & 0.62 & 0.34 & 0.36 \\
Intervalo de confiança & $0.45-0.75$ & $0.14-0.51$ & $0.19-0.51$ \\
\hline
\end{tabular}

Tabela 4

Análise do coeficiente de concordancia de Lin (Rc) TAG x TCDP

\begin{tabular}{lccc}
\hline & Grupo 1 & Grupo 2 & Grupo 3 \\
\hline $\mathrm{R}_{\text {c }}$ & 0.45 & 0.22 & 0.45 \\
Intervalo de confiança & $0.31-0.57$ & $0.08-0.35$ & $0.25-0.61$ \\
\hline
\end{tabular}

Tabela 6

Análise do coeficiente de Lin (Rc) IC x TCDP

\begin{tabular}{lccc}
\hline & Grupo 1 & Grupo 2 & Grupo 3 \\
\hline $\mathbf{R}_{\text {c }}$ & 0.64 & 0.50 & 0.26 \\
Intervalo de confiança & $0.48-0.75$ & $0.30-0.66$ & $0.05-0.45$ \\
\hline
\end{tabular}

Tabela 3

Análise de Bland\&Altman para os diferentes grupos

\begin{tabular}{lccc}
\hline & Grupo 1 & Grupo 2 & Grupo 3 \\
\hline Média & -2.1 & -2.5 & -4.5 \\
Desvio Padrão & \pm 2.67 & \pm 2.9 & \pm 3.18 \\
\hline
\end{tabular}

Tabela 5

Análise de Bland\&Altmann para os diferentes grupos

\begin{tabular}{lccc}
\hline & Grupo 1 & Grupo 2 & Grupo 3 \\
\hline Média & -3.6 & -3.4 & -2.2 \\
Desvio Padrão & \pm 1.96 & \pm 2.39 & \pm 2.74 \\
\hline
\end{tabular}

Tabela 7

Análise de Bland\&AItman IC x TCDP

\begin{tabular}{lccc}
\hline & Grupo 1 & Grupo 2 & Grupo 3 \\
\hline Média & -1.6 & -0.9 & -2.2 \\
Desvio Padrão & \pm 2.54 & \pm 2.5 & \pm 3.75 \\
\hline
\end{tabular}


utilizando-se o modelo de Line o de Bland \& Altman, sendo calculados a média das diferenças entre os métodos (viés) e intervalos de confiança $95 \%$ para os limites de concordância ${ }^{(9-10)}$.

$O$ coeficiente de concordância de Lin $\left(R_{c}\right)$ foi empregado para determinar se as medidas obtidas entre os tonômetros desviavam-se significativamente da linha de perfeita concordância (linha de 45 graus com origem no 0 dos eixos x e y). Excelente concordância foi definida como $\mathrm{R}_{\mathrm{c}}>0.90$, satisfatório como $\mathrm{R}_{\mathrm{c}} 0.6-0.9 \mathrm{e}$ insatisfatório como $\mathrm{R}_{\mathrm{c}}<0.6$.

No gráfico de Bland \& Altman as diferenças entre as medidas de dois aparelhos foram traçadas contra os valores médios da PIO encontrados por cada tonômetro. A média das diferenças é representada por uma linha horizontal continua no diagrama, sendo que as linhas horizontais pontilhadas correspondem aos limites superiores e inferiores de concordância, calculados pelo desvio padrão das diferenças.

A análise estatística dos dados foi efetuada utilizando-se $S A S$ versão 9.1 (Statistical Analysis System, Cary, NC, USA).

\section{Goldmann x ICare ${ }^{\circledR}$ :}

Entre o TAG e o IC, quando todos os grupos foram analisados em conjunto, observou-se um coeficiente de concordância de Lin ( $\mathrm{R}_{\mathrm{c}}$ ) de 0.47 (intervalo de confiança de $95 \%$ de 0.37 - 0.56) (Figura 1). Quando observados nos grupos 1,2 e 3, os coeficientes de concordância de Lin $\left(R_{c}\right)$ foram respectivamente: 0.62 (intervalo de confiança de $95 \%$ de 0.45 - 0.75); 0.34 (intervalo de confiança de $95 \%$ de $0.14-0.51$ ) e 0.36 (intervalo de confiança de $95 \%$ de 0.19 - 0.51) (Tabela 2).

$\mathrm{Na}$ análise de Bland \& Altman a média das diferenças (TAG - IC) da PIO entre os tonômetros foi de -2.9 ( \pm 3.07 ) (Figura 2). Na análise entre os grupos 1, 2 e 3, os valores encontrados foram respectivamente: $-2.1( \pm 2.67)$; $-2.5( \pm 2.9)$ e $-4.5( \pm 3.18)$ (Tabela 3).

\section{Goldmann x Pascal ${ }^{\oplus:}$}

Entre o TAG e o TCDP, quando todos os grupos foram analisados em conjunto, observou-se um coeficiente de concordância de Lin $\left(\mathrm{R}_{\mathrm{c}}\right)$ de 0.38 (intervalo de confiança de $95 \%$ de 0.29 - 0.46) (Figura 3). Quando observados nos grupos 1, 2 e 3, os coeficientes de concordância de Lin ( $\left.\mathrm{R}_{\mathrm{c}}\right)$ foram respectivamente: 0.45 (intervalo de confiança de $95 \%$ de $0.31-0.57$ ); 0.22 (intervalo de confiança de $95 \%$ de 0.08 - 0.35) e 0.45 (intervalo de confiança de $95 \%$ de 0.25 - 0.61) (Tabela 4).

$\mathrm{Na}$ análise de Bland \& Altman a média das diferenças (TAG - TCDP) da PIO entre os tonômetros foi de

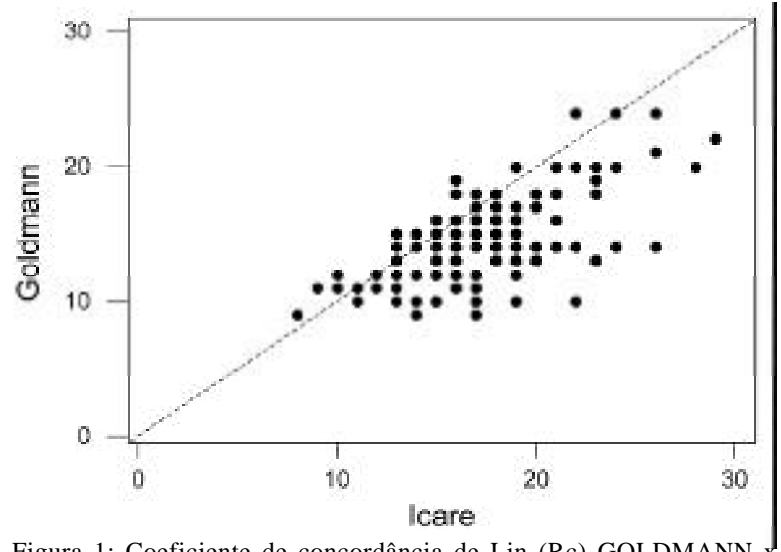

Figura 1: Coeficiente de concordância de Lin (Rc) GOLDMANN x ICARE

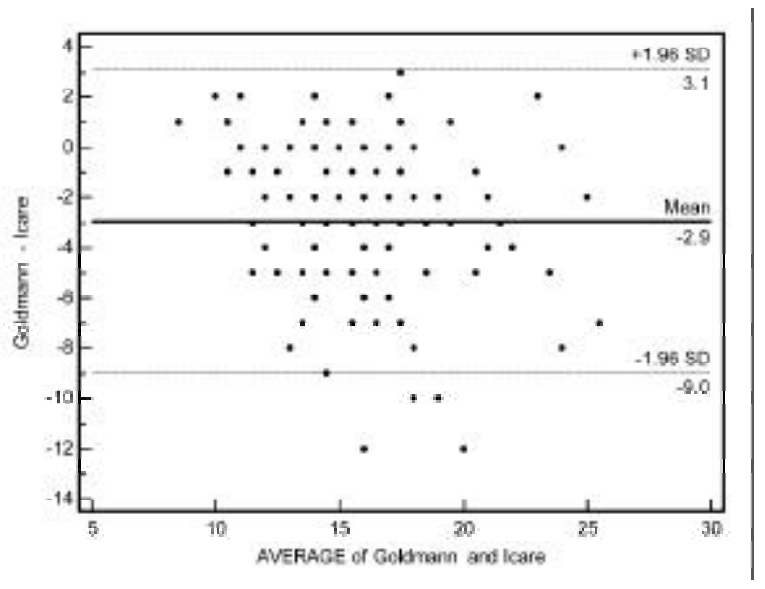

Figura 2: Gráfico de Bland\&Altman GOLDMANN x ICARE

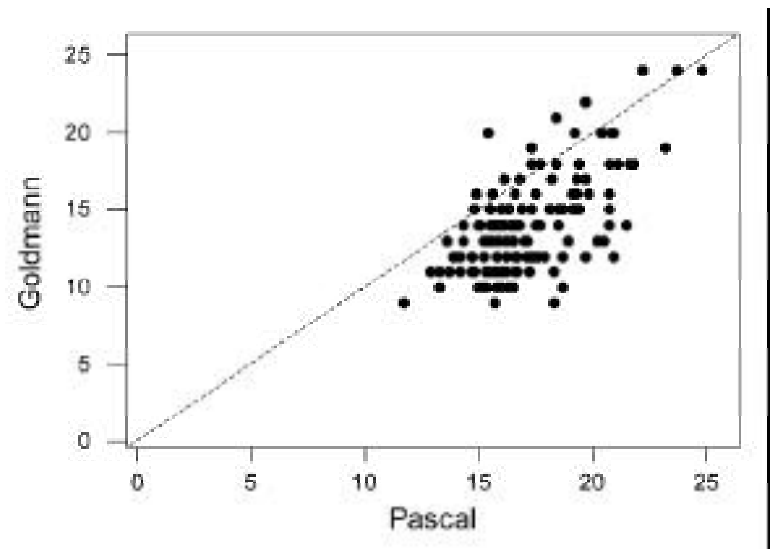

Figura 3: Coeficiente de concordância de Lin (Rc) GOLDMANN x PASCAL 


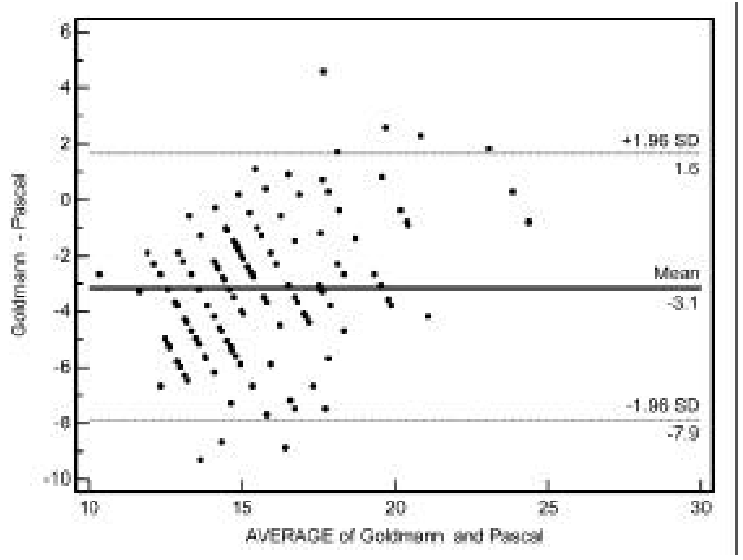

Figura 4: Gráfico de Bland\&Altman GOLDMANN x PASCAL

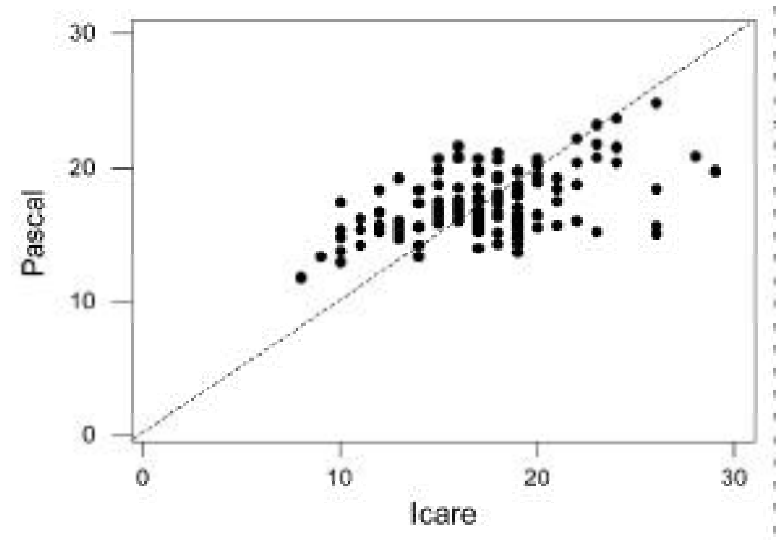

Figura 5: Coeficiente de concordância de Lin (Rc)

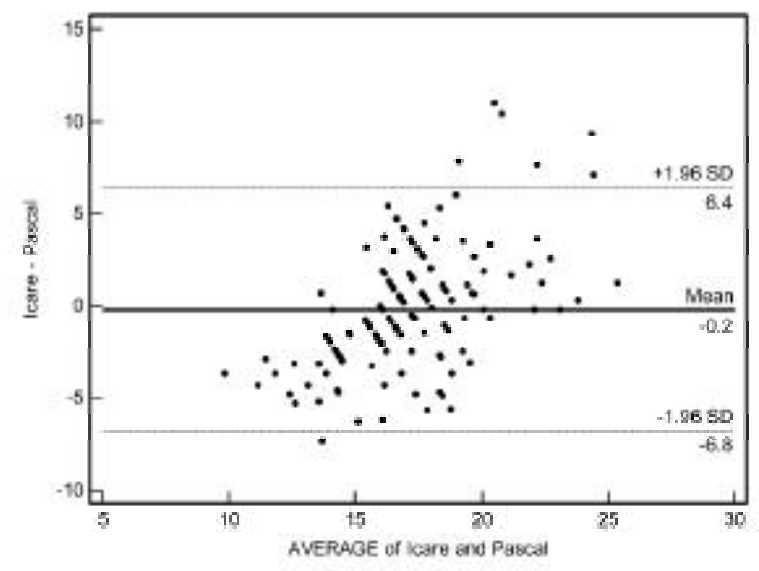

Figura 6: Gráfico de Bland\&Altman IC x TCDP
-3.1 ( \pm 2.42 ) (Figura 4). Na análise entre os grupos 1, 2 e 3 , os valores encontrados foram respectivamente: -3.6 $( \pm 1.96) ;-3.4( \pm 2.39)$ e $-2.2( \pm 2.74)$ (Tabela 5).

\section{ICare $^{\circledast} \times$ Pascal $^{\oplus}$ :}

A PIO média obtida pelo TCDP foi $17.3 \mathrm{mmHg}( \pm$ 2.4) e pelo IC, $17.1 \mathrm{mmHg}( \pm 4)$ valores considerados semelhantes. Entretanto, quando todos os grupos foram analisados em conjunto, observou-se um coeficiente de concordância de Lin $\left(\mathrm{R}_{\mathrm{c}}\right)$ de 0.46 (intervalo de confiança de 95\% de 0.34 - 0.56) (Figura 5). Quando analisados separadamente (grupos 1,2 e 3), os coeficientes de concordância de $\operatorname{Lin}\left(\mathrm{R}_{\mathrm{c}}\right)$ foram respectivamente: 0.64 (intervalo de confiança de $95 \%$ de $0.48-0.75$ ); 0.50 (intervalo de confiança de $95 \%$ de $0.30-0.66$ ) e 0.26 (intervalo de confiança de $95 \%$ de $0.05-0.45$ ) (Tabela 6).

$\mathrm{Na}$ análise de Bland \& Altman a média das diferenças (IC - TCDP) da PIO entre os tonômetros foi de $0.2( \pm 3.35)$ (Figura 6). Na análise entre os grupos 1, $2 \mathrm{e}$ 3 , os valores encontrados foram respectivamente: -1.6 $( \pm 2.54) ;-0.9( \pm 2.50)$ e $-2.2( \pm 3.75)$ (Tabela 7$)$.

\section{Dıscussão}

A determinação precisa da PIO é fundamental em qualquer exame oftalmológico. Diversos métodos foram utilizados no passado para medir a PIO. Atualmente, novos tonômetros, baseados em diferentes princípios, estão sendo continuamente desenvolvidos ${ }^{(1)}$.

A medida da ECC tornou-se um importante fator biométrico e é parte essencial na propedêutica do glaucoma $^{(3.5,11-12)}$.

Os valores da PIO obtidos com o TCDP em nosso estudo foram maiores que os encontrados com o TAG ${ }^{(3,4,11)}$. A média de diferença entre as duas técnicas foi de -3.1 $( \pm 2.42)$. Quando analisados de acordo com a ECC, a concordância entre os aparelhos foi melhor em pacientes com ECC menor que $520 \mu \mathrm{m}$ e maior que $560 \mu \mathrm{m}$. Um considerável desacordo entre os dois instrumentos tem como possíveis influências a diferença de calibração, influência de ECC no TAG e propriedades corneanas ${ }^{(6)}$. Para uso clínico, a leitura mais alta da PIO no TCDP deve ser considerada. Entretanto, as medidas como TCDP parecem não depender da ECC, corroborando alguns trabalhos ${ }^{(24,1,1,1-1-14)}$.

Brusini e cols. demonstraram uma razoável concordância entre as leituras da PIO entre o IC e o TAG, porém, com valores variáveis de acordo com a ECC, sendo que o IC forneceu valores maiores de PIO que o $\mathrm{TAG}^{(1)}$. Em nosso estudo, há uma pequena diferença, mas estatisticamente significativa entre as medidas da PIO 
obtidas com o IC e com o TAG, com média das diferenças de $-2.9( \pm 3.07)$. A concordância das leituras da PIO entre os tonômetros foi satisfatória somente em pacientes que apresentavam ECC menor que $520 \mu \mathrm{m}$.

Na comparação da PIO obtida entre os aparelhos, a melhor concordância foi observada entre os tonômetros IC e TCDP, com média das diferenças (IC - TCDP) de $0.2( \pm 3.35)$. Observou-se uma concordância baixa em pacientes com ECC maior que $560 \mu \mathrm{m}$, o que pode ser explicado pelo fato do IC ser influenciado pela ECC ao contrário do $\operatorname{TCDP}^{(1,3-4,6-7,11)}$. Até a presente data, não foram encontrados na literatura compulsada estudos que avaliassem concomitantemente IC, TAG e TCDP.

\section{Conclusão}

Existe uma diferença estatisticamente significante entre os valores da PIO obtidos com o IC, o PCDT e o TAG, quando relacionados com a medida da ECC, ocorrendo uma melhor concordância entre estes valores nas córneas de menor espessura.

\section{Abstract}

Purpose: Could the central corneal thickness (CCT) change the intraocular pressure (IOP) measured by ICARE (IC) GOLDMANN APPLANATIONTONOMETER(GAT) and PASCAL DYNAMIC COUNTOUR TONOMETER (PDCT). Methods: Before using the anesthesia we have measured the IOP with IC tonometer. After that we have used topical anesthesia to obtain the CCT using an ultrasound pachymeter (Mentor Co.) and the IOP measured by PCDT followed by a drop of fluorescein to measured IOP with GAT. Results: 138 eyes of 71 patients were analyzed. Patients were divided into 3 groups according to CCT. Best correlation was observed with IC and PDCT, showing a mean average difference of -0.2. The intraocular pressure data obtained from PDCT showed higher values when compared with $G A T$, with a mean average difference of -3.1. There are also a statistical significant difference with the IOP measurements with IC and GAT. Conclusion: There are a significant difference in IOP measurements obtained by IC, GAT and PCDT when compared with CCT.

Keywords: Tonometry, ocular/methods; Corneal anatomy \& histology; Glaucoma; Intra-ocular pressure

\section{RefERÊNCIAS}

1. Brusini P, Salvetat ML, Zeppieri M, Tosoni C, Parisi L. Comparison of ICare tonometer with Goldmann applanation tonometer in glaucoma patients. J Glaucoma. 2006; 15(3):213-7.
2. Kanngiesser HE, Kniestedt C, Robert YC. Dynamic contour tonometry: presentation of a new tonometer. J Glaucoma. 2005; 14(5):344-50.

3. Schneider E, Grehn F. Intraocular pressure measurementcomparison of dynamic contour tonometry and goldmann applanation tonometry. J Glaucoma. 2006; 15(1):2-6. Comment in: J Glaucoma. 2006; 15(5):471.

4. Kniestedt C, Lin S, Choe J, Nee M, Bostrom A, Stürmer J, Stamper, RL. Correlation between intraocular pressure, central corneal thickness, stage of glaucoma, and demographic patient data: prospective analysis of biophysical parameters in tertiary glaucoma practice populations. J Glaucoma. 2006; 15(2):91-7.

5. Iliev ME, Goldblum D, Katsoulis K, Amstutz C, Frueh B. Comparison of rebound tonometry with Goldmann applanation tonometry and correlation with central corneal thickness. Br J Ophthalmol. 2006; 90(7):833-5.

6. García-Resúa C, González-Meijome JM, Gilino J, YebraPimentel E. Accuracy of the new ICare rebound tonometer vs. other portable tonometers in healthy eyes. Optom Vis Sci. 2006; 83(2):102-7.

7. Kotecha A, White ET, Shewry JM, Garway-Heath DF. The relative effects of corneal thickness and age on Goldmann applanation tonometry and dynamic contour tonometry. $\mathrm{Br} \mathbf{J}$ Ophthalmol. 2005; 89(12):1572-5.

8. Herndon LW. Measuring intraocular pressure-adjustments for corneal thickness and new technologies. Curr Opin Ophthalmol. 2006; 17(2):115-9.

9. Bland JM, Altman DG. Statistical methods for assessing agreement between two methods of clinical measurement. Lancet. 1986; 1(8476): 307-10.

10. Lin LI. A concordance correlation coefficient to evaluate reproducibility. Biometrics. 1989; 45(1):255-68.

11. Kniestedt C, Lin S, Choe J, Bostrom A, Nee M, Stamper RL. Clinical comparison of contour and applanation tonometry and their relationship to pachymetry. Arch Ophthalmol. 2005;123(11):1532-7.

12. Kaufmann C, Bachmann LM, Robert YC, Thiel MA. Ocular pulse amplitude in healthy subjects as measured by dynamic countour tonometry. Arch Ophthalmol. 2006; 124(8):1104-8.

13. Brown KE, Congdon NG. Corneal structure and biomechanics: impact on the diagnosis and management of glaucoma. Curr Opin Ophthalmol. 2006; 17(4):338-43.

14. Browning AC, Bhan A, Rotchford AP, Shah S, Dua HS. The effect of corneal thickness on intraocular pressure measurement in patients with corneal pathology. $\mathrm{Br} \mathrm{J}$ Ophthalmol. 2004; 88(11):1395-9.

\section{ENDEREÇO PARA CORRESPONDÊNCIA: \\ Av.lbirapuera, $n^{\circ} 2907$ - Conj. 1601 \\ CEP 04029-200 - São Paulo - SP}

Email: clrehder@yahoo.com.br 